\title{
Bonding Metal Brackets on Tooth Surfaces
}

\author{
Breuning $\mathrm{KH}^{1^{*}}$, Dekkers $\mathrm{L}^{3}$, Beeftink $\mathrm{M}^{3}$, Schols $\mathrm{JH}^{1}$ and Wolke $\mathrm{J}^{2}$ \\ ${ }^{1}$ Department of Orthodontics and Craniofacial Biology, Radboud Medical Centre, Nijmegen, the Netherlands \\ ${ }^{2}$ Department of Periodontology and Biomaterials, Radboud Medical Centre, Nijmegen, the Netherlands \\ ${ }^{3}$ Department of Dentistry, Radboud Medical Centre, Nijmegen, the Netherlands
}

"Corresponding author: K.H. Breuning, Department of Orthodontics and Craniofacial Biology, Radboud University Nijmegen, Medical Centre, PO Box 9101, 6500HB Nijmegen, The Netherlands, Tel: +31243617204; E-mail: hero.breuning@radboudumc.nl

Rec date: Mar 11, 2014, Acc date: Apr 17, 2014, Pub date: Apr 19, 2014

Copyright: $\odot 2014$ Breuning $\mathrm{H}$, et al. This is an open-access article distributed under the terms of the Creative Commons Attribution License, which permits unrestricted use, distribution, and reproduction in any medium, provided the original author and source are credited.

\begin{abstract}
Objectives: To test the effect of different surface preparation methods on accurate bonding of orthodontic brackets on enamel, composite, and ceramic surfaces. In addition, we evaluated differences in bonding force and the adhesive remaining between precoated and non-precoated brackets after bonding on these surfaces.

Methods: Samples of enamel, composite, and ceramic surfaces were prepared for bonding orthodontic brackets using roughening with a bur, a sandblaster, phosphoric acid solution, etching with hydrofluoric acid solution, and a porcelain activator depending on the surface tested. After surface preparation, the roughness of the surface was evaluated. Then, a primer was applied and precoated and non-precoated brackets were bonded to the surface. The bond strength was tested and the amount of remaining adhesive was evaluated.

Results: Thermo cycling of teeth did not result in differences in bond strength. There was a significant increase in bonding strength between brackets without adhesive and precoated brackets after bonding on sandblasted composite surfaces. An increased roughness of dental surfaces did not result in increased bonding strength. There was adequate bonding of metal brackets on enamel after etching with $37 \%$ phosphoric acid for 30 seconds. Enamel surfaces that were roughened with a drill or sandblaster before etching had shear bonding strengths over the recommended $8 \mathrm{MPa}$. Just roughening of the enamel surface did not lead to efficient bonding. On a composite surface, roughening with a bur led to adequate bonding; while, sandblasting did not. For ceramics, after etching with hydrofluoric acid a porcelain activator was required. A reduction in surface roughness led to an increase in bond strength. After debonding, the quantity of adhesive remaining depended on the surface properties and the surface preparation before bonding.
\end{abstract}

Clinical Significance: Bracket failure during orthodontic treatment leads to increased treatment time and costs. Bonding forces should not be too low to prevent bracket failure or too high to prevent damage to the surface from debonding. Both precoated and non-precoated bracket types can be used for adequate bonding on the tested dental surfaces.

Keywords: Bonding; Brackets; Enamel; Composite; Ceramics

\section{Introduction}

For decades, bonding of orthodontic brackets and tubes has replaced traditional banding. More adult patients now request orthodontic treatments; thus, effective bonding on restored tooth surfaces, such as composites and porcelain, is required. The failure rate of bonding brackets on different tooth surfaces can be reduced if proper bonding protocols are used. For adequate bonding on teeth, the brackets should be able to withstand the orthodontic forces and the forces acting on the brackets during biting and chewing. According to Reynolds [1], shear bond strength values for adequate bonding should be between 5.9 to 7.8 Megapascal (MPa). High values of shear bond strength could lead to enamel damage during removal of the brackets and low values will lead to bond failures. Successful bonding on tooth surfaces requires preparation of the surface for proper mechanical or chemical bonding, adequate bracket base design, and a proper adhesive. After orthodontic treatment, the brackets and tubes should be easy removed from the tooth, without damage to its surface.
Restoration of the esthetics of the tooth surface after bracket removal should be easy to achieve.

Several bonding protocols for effective bonding on enamel, composite, and ceramic surfaces have been introduced, but there is no agreement on the most effective procedure [2-6]. Precoating of orthodontic brackets (with adhesive on the bracket mesh pad) was introduced to reduce the number of operations needed for bonding as well as bonding time and bond failures (APC coated brackets, Unitek/3M, Monrovia, California, US). If precoated brackets are used, they should be stored in dark containers and the adhesive of these brackets should be cured using a dental light. Removal of adhesive flush around the bracket base is necessary for easier cleaning, and less white spot formation around the bracket base can be expected. Prior studies reported conflicting results when using precoated brackets on different tooth surfaces $[7,8]$. In this study, the accuracy of different procedures for bonding traditional metal brackets and precoated orthodontic brackets on different tooth surfaces was evaluated. 


\section{Materials and methods}

For this study, the surfaces of samples of extracted teeth (upper premolars, lower first premolars), without restorations on the planned position for orthodontic brackets, were used. The teeth were placed in $0.1 \%$ thymol solution and stored in distilled water at room temperature, which was replaced on a regular basis to prevent deterioration. In a randomly selected sample of the extracted premolars, composite restorations were made into intact buccal surfaces. A dental preparation with a depth of $1 \mathrm{~mm}$ was made with a cylindrical diamond bur using a water spray for cooling. Undercuts in this preparation were made with a solid carbide drill for retention of the composite. The outline of the preparation was at least $1 \mathrm{~mm}$ larger than the dimension of the selected orthodontic bracket. After cleaning and drying, the preparation was etched with a $37 \%$ phosphoric agent (Etching gel, Medium Viscosity, DMG Chemisch-Pharmazeutische Fabrik, Hamburg, Germany) for 10 seconds. After removing the etching gel with water and air spray, the preparation was dried. A thin layer of primer was then applied (Clearfil Photobond, Kuraray Noritake Dental Inc. Otemachi, Chiyoda, ku, Tokyo,Japan), dried with clean air for 5 seconds, and cured for 10 seconds according to the instruction manual with a dental curing light (Poly lux 2, KaVo Dental $\mathrm{GmbH}$, Warthausen, Germany) at a wave length of 420-480 $\mathrm{nm}$ and an intensity of $400 \mathrm{~W} / \mathrm{cm}^{2}$. The preparation was then completely filled with a posterior hybrid composite (Clearfil Photo Posterior, Kuraray Noritake Dental Inc. Otemachi, Chiyoda, ku, Tokyo, Japan). Before curing, the surface of the restoration was modeled with an instrument. The composite was then light cured with the same device at a distance of $5 \mathrm{~mm}$ for 20 seconds, polished with pumice, cleaned, and dried.

After this procedure, the selected extracted premolars were kept in water at room temperature $\left( \pm 20^{\circ} \mathrm{C}\right)$. In the dental laboratory, a set of samples with a ceramic surface (GC initial LF, GC Europe n.v., Leuven, Belgium) and a convexity similar to that of a central upper incisor, was made on a metal layer (Wiron 99, Bego, Bremer Goldschlägerei Wilh. Herbst GmbH \& Co. KGHerbst GmbH \& Co KG, Wilhelm-Herbst-Str. Bremen, Germany) and kept in water at room temperature. At least 10 samples were available for testing. GermanyBefore bonding the brackets, different surface preparation methods were used to increase the surface roughness.

A: roughening with a Carborundum bur (666/025, medium Meisinger, Neuss, Germany). The bur was inserted in a slow speed dental hand piece and moved three times, from left to right in parallel and in contact with the dental surface.

B: Roughening with a sandblaster (Microcab ${ }^{\text {tw }}$, Danville Materials, San Ramon, California, USA) containing aluminum-oxide particles, with a dimension of $50 \mu \mathrm{m}$, in a direction perpendicular to the dental surface for 10 seconds at a distance of $25 \mathrm{~mm}$.

C: The surface was covered with a $9 \%$ hydrofluoric acid solution (PorceLock $\odot$, Porcelain Etching Solution, Den-Mat Corporation, Santa Maria, U.S.A.) for 120 seconds.

D: Etching of the surface with an etching gel containing 37\% phosphoric acid (Unitek Etching Gel System 3M Unitek, Orthodontic Products, Monrovia, U.S.A.) for 30 seconds.

E: Salinization of the (ceramic) dental surface with a porcelain activator (Clearfil, Porcelain Bond Activator 3M Unitek, Orthodontic Products, Monrovia, U.S.A.).

After applying one or several of these surfaces preparation methods (A-E), the surfaces were cleaned with a spray (water and air). The roughness of the dental surface was tested after each surface preparation method using 5 randomly chosen samples. For testing the surface roughness, a stylus (diamond, $60^{\circ}$ ) of the Universal Surface Tester (UTS) was used over a distance of $4 \mathrm{~mm}$ and the roughness value $(\mathrm{Ra})$ of the tested surface was recorded. As this testing method is not destructive, these samples could be used for further tests. After testing the roughness of the surfaces, all samples were thoroughly dried, with a clean air flow. Then, a thin layer of a liquid resin primer (Transbond $^{\text {is }}$ XT primer, 3M Unitek, Orthodontic Products, Monrovia, U.S.A.) was applied with a small foam pellet on the enamel and composite surfaces according to the manual of the primer. The resin of the primer penetrates the irregularities in the enamel and improves the locking of the adhesive to the dental surface. For a randomly chosen sample of ceramic test surfaces, Porcelain Bond Activator (Clearfil, Porcelain Bond Activator, Kuraray Noritake Dental Inc. Otemachi, Chiyoda, ku, Tokyo, Japan), was used according to the manual of the primer (surface treatment method E). To evaluate differences in mean bonding strength and the adhesive remnant (ARI) scores, precoated and non-precoated brackets (Victory Series $^{\mathrm{m}}$, Low Profile, 3M Unitek, Orthodontic Products, Monrovia, U.S.A.), with a pre-shaped bracket base for premolars and central upper incisors, and adhesive (Transbond ${ }^{n}$ PLUS Color Change Adhesive, 3M Unitek, Orthodontic Products, Monrovia,U.S.A.) were used in this study. The APC adhesive used in this study contained composite resins formulated from glass particles and dimethacrylate monomers and provides clinically acceptable shear bond forces for metal brackets [9]. The selected adhesive has sufficient viscosity so that the bonded attachments do not drift away from their desired position before the adhesive is cured. Orthodontic adhesive was applied to the mesh pad of a sample of non-precoated metal brackets. Then, the bracket was positioned on the prepared dental surface at the desired position for bonding orthodontic brackets. After positioning, the bracket was firmly pushed onto the surface to ensure a close contact between the bracket base, the adhesive, and the prepared surface. Excessive adhesive flash around the bracket base was removed with a plastic filling instrument before curing the adhesive. A second sample of the same Victory Series ${ }^{\text {ma }}$ Low Profile brackets, prepasted with adhesive (Transbond ${ }^{m m}$ APC $^{m m}$ Plus 3M Unitek, Orthodontic Products, Monrovia, U.S.A.), was kept in a special individual bracket kit to prevent curing of the adhesive before bracket bonding. After removal from the bracket kit, the precoated bracket was immediately positioned on the prepared surface using the same bracket positioning procedure. Directly after positioning the brackets and removal of excessive adhesive flash, the adhesive was cured with the dental adhesive LED curing device as described earlier. During curing, the tip of the curing light was held at a $45^{\circ}$ angle relative to the surface in contact with the bracket. The curing time was 10 seconds at the mesial-distal side of the bracket base. After adhesive curing, the samples were stored in water at room temperature $\left( \pm 20^{\circ} \mathrm{C}\right)$ for 2 days.

Some samples were evaluated in a thermo cycling machine (Haake W 153 M Thermo Electron, Karlsruhe, Germany) for 30 seconds in water with temperatures of $5^{\circ} \mathrm{C}$ and $55^{\circ} \mathrm{C}$. This thermo cycling procedure was repeated 5000 times, corresponding to an oral environment of about half a year. After thermo cycling, the samples were secured in a metal cylinder with a blend of synthetic resin and alpha gypsum (Resin Rock ${ }^{\mathrm{rm}}$, Whip Mix ${ }^{\oplus}$ Company, Louisville, USA) to be used in the bond strength testing device (MTS 858 Mini Bionix II, Eden Prairie, USA) with a load capacity of $15 \mathrm{kN}$ and torque capacity of $150 \mathrm{Nm}$, and a displacement range of $+/-50 \mathrm{~mm}$ to evaluate the bond shear strength. The speed of the measuring stylus was set to 0.5 
Page 3 of 6

$\mathrm{mm} /$ minute. The maximal force was recorded in Newtons $(\mathrm{N})$ and converted to Megapascals (Mpa) by dividing the maximum force value by the total surface area of the brackets. The total surface area of the premolar bracket base used was $13.8 \mathrm{~m}^{2}$ and the surface area of the incisor bracket base was $14.91 \mathrm{~m}^{2}$.

After release of the bonding between the dental surface and the bracket, the remaining adhesive on the dental surface was evaluated with a stereo microscope (Leica, V50x, Wetzlar, Germany). Each tooth surface was photographed with the Infranview program Version 4.0 (Irfan Skiljan,Wiener Neustadt, Austria). For each sample, the adhesive remnant index (ARI) scores were recorded according to the original description of Årtun and Bergland [10-12], with the following scale: 0 , no adhesive left on the tooth; 1 , less than half of the adhesive left on the tooth; 2, more than half of the adhesive left on the tooth; and 3, all adhesive left on the tooth, with a distinct impression of the bracket mesh. In each group, 10 samples were tested. During the testing procedure, some samples could not be used because of breakage of the tooth or the connection between the tooth and the fixation medium. These samples were excluded from the study. For all groups tested in this study, there were sufficient samples for statistical evaluation.

\section{Statistical evaluation}

The roughness of the different prepared dental surfaces was compared using the Student-t-test and variance analysis, provided that the measurements were normally distributed. The effect of thermo cycling on the shear bond strength was evaluated with the Student, Ttest. The differences in mean shear strength and adhesive remnant indexes of brackets bonded on differently prepared tooth surfaces were evaluated with the Student, T-test. Finally, differences in the mean shear bond strength of non prepasted and prepasted brackets bonded on differently prepared tooth surfaces were evaluated with the Student, T-test. For each statistical analysis, the significance level was set to $\mathrm{p}<0.05$.

\section{Results}

Before thermo cycling and bonding of the brackets, the roughness of the enamel and composite surfaces was evaluated after different surface preparation methods. Significant differences were found. For the enamel, roughening with a bur resulted in a significantly rougher surface compared with a sandblaster $(\mathrm{p}<0.05)$ and etching with phosphoric acid $(\mathrm{p}<0.001)$. Using a bur did not result in significantly different roughening on enamel vs. composite surface. When a sandblaster was used, enamel surfaces were significantly less rough, compared with composite $(\mathrm{p}<0.001)$ and ceramic $(\mathrm{p}<0.01)$ surfaces, which were not statistically different from each other. The difference in surface roughness after etching enamel with phosphoric acid or ceramic surfaces with hydrofluoric acid was not statistically different. The roughness after different surface preparation methods is presented as a histogram in Figure 1.

\section{Surface treatment method}

\section{1=Enamel, 2 = Composite, $3=$ Ceramic.}

$\mathrm{A}=$ Roughening with a drill, $\mathrm{B}=$ Roughening with a sandblaster, $\mathrm{C}=$ Etching with hydrofluoric acid, $\mathrm{D}=$ Etching with phosphoric acid, $\mathrm{E}=$ Salinization as description of the methods used.
The effect of thermo cycling on the mean shear bond strength (in $\mathrm{MPa}$ ) of brackets bonded to enamel after roughening with a bur (A), sandblasting (B), or etching with phosphoric acid (D) followed by cleaning and drying was evaluated using 5 randomly selected samples. No significant differences in shear bond strength were found. The effect of thermo cycling on the adhesive remaining index (ARI) of brackets bonded on enamel after roughening with a drill (A), sandblasting (B), or etching with phosphoric acid (D) was evaluated using 10 randomly selected samples. No significant differences were found. The differences in mean shear bond strength after the different surface preparation methods for the 3 different materials are shown in Table 1.

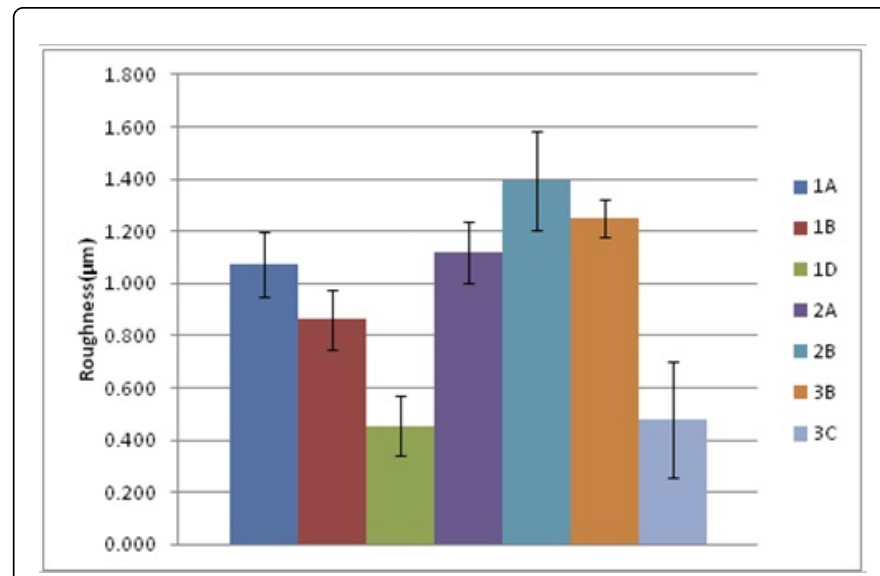

Figure 1: Roughness after different surface preparation methods

\begin{tabular}{|c|c|c|c|c|c|c|}
\hline Group & Mean(SD) & Group & Mean(SD) & Group & Mean(SD) & Significance \\
\hline \multirow{3}{*}{$1 \mathrm{~A}$ pre } & \multirow{3}{*}{$\begin{array}{l}1.218(0.81 \\
33)\end{array}$} & \multirow{3}{*}{$1 \mathrm{~B}$ pre } & \multirow{3}{*}{$\begin{array}{l}2.357(1.21 \\
0)\end{array}$} & \multirow{3}{*}{ 1D not } & \multirow{3}{*}{$\begin{array}{l}5.817(3.94 \\
9)\end{array}$} & $1 A+1 B$ ns \\
\hline & & & & & & $\begin{array}{l}1 A+1 D \\
p<0.01\end{array}$ \\
\hline & & & & & & $\begin{array}{l}1 B+1 D \\
p<0.05\end{array}$ \\
\hline \multirow{3}{*}{$1 \mathrm{~A}$ not } & \multirow{3}{*}{$\begin{array}{l}1.235(0.57 \\
84)\end{array}$} & \multirow{3}{*}{$1 \mathrm{~B}$ not } & \multirow{3}{*}{$\begin{array}{l}1.347(0.57 \\
84)\end{array}$} & \multirow{3}{*}{ 1D not } & \multirow{3}{*}{$\begin{array}{l}8.276(5.67 \\
0)\end{array}$} & $1 \mathrm{~A}+1 \mathrm{~B} n \mathrm{~s}$ \\
\hline & & & & & & $\begin{array}{l}1 A+1 D \\
p<0.05\end{array}$ \\
\hline & & & & & & $\begin{array}{l}1 B+1 D \\
p<0.01\end{array}$ \\
\hline $2 \mathrm{~A}$ pre & $\begin{array}{l}6.018(3.64 \\
7)\end{array}$ & 2B pre & $\begin{array}{l}5.360(3.78 \\
7)\end{array}$ & & & ns \\
\hline $2 \mathrm{~A}$ not & $\begin{array}{l}\text { 6.395(3.31 } \\
\text { 9) }\end{array}$ & 2B not & $\begin{array}{l}2.459(1.47 \\
2)\end{array}$ & & & $p<0.01$ \\
\hline $\begin{array}{l}3 B E \\
\text { not }\end{array}$ & $\begin{array}{l}1.267(0.97 \\
43)\end{array}$ & $\begin{array}{l}3 \mathrm{CE} \\
\text { not }\end{array}$ & $\begin{array}{l}7.090(3.42 \\
5)\end{array}$ & & & $p<0.001$ \\
\hline
\end{tabular}

Table 1: Differences in mean shear bond strength after the different surface preparation methods

The differences in mean shear bond strength between precoated brackets and non precoated brackets after different preparation methods for enamel and composite surfaces were also evaluated. The mean shear bond strength of precoated brackets was significantly 
Page 4 of 6

$(\mathrm{p}<0.05)$ higher only for composite surfaces prepared with a sandblaster. The mean shear bond strengths of brackets bonded to enamel, composite, and ceramic surfaces after different surface preparation methods are presented in Figure 2.

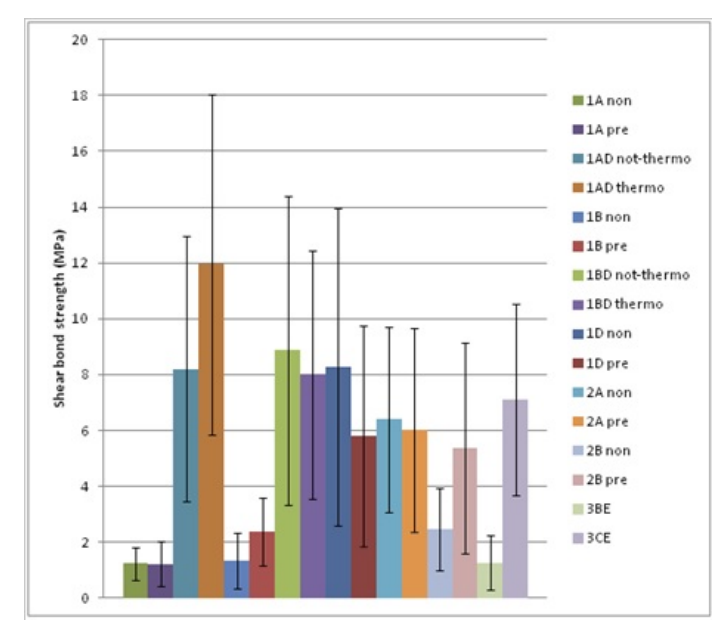

Figure 2: Mean shear bond strengths of brackets bonded to enamel, composite, and ceramic surfaces after different surface preparation methods

Differences in the mean ARI-scores after accurate bonding (shear bond strength over $5.7 \mathrm{MPa}$ ) between non precoated and precoated brackets on enamel, composite, and ceramic surfaces were evaluated after different surface preparation methods. For enamel, we found significantly more adhesive on the surface after bracket removal, compared with the mean ARI for composite and ceramic surfaces $(p<0.01)$. The mean ARI scores of brackets bonded to enamel, composite, and ceramic surfaces after different surface preparation methods are presented in Figure 3.

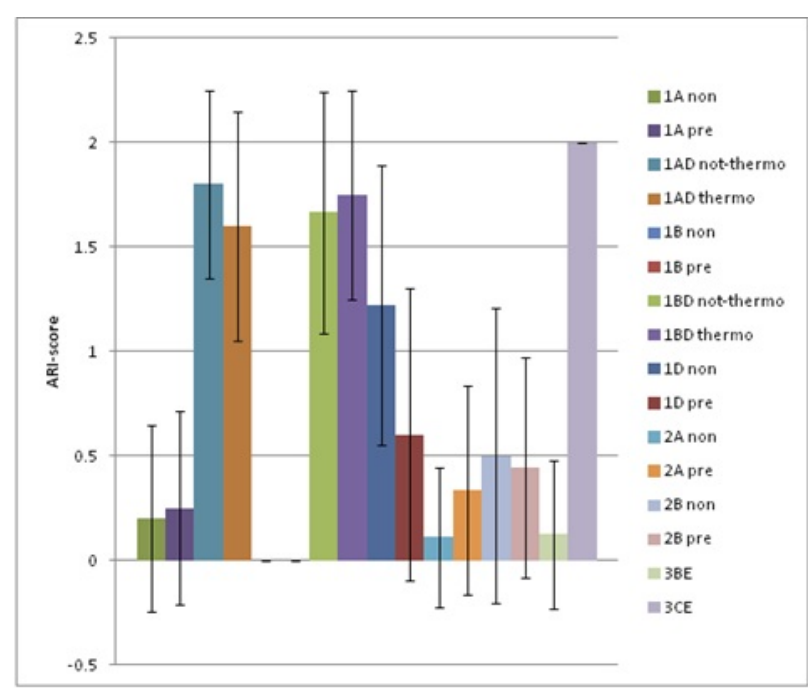

Figure 3: Mean ARI scores of brackets bonded to enamel, composite, and ceramic surfaces after different surface preparation methods
No significant differences were found for the mean ARI-scores for precoated and non precoated brackets bonded on enamel after different methods of surface preparation.

\section{Discussion}

Before surface preparation, the dental surfaces of the extracted premolars were not cleaned using a mix of pumice and water, a prophylaxis paste, a rubber cup, or a polishing brush mounted on a low-speed rotary-instrument. Several studies demonstrated that prior pumicing of enamel has no significant effect on bond strength for fixation of orthodontic brackets on enamel surfaces [13-15]. Conditioning tooth surfaces for bonding orthodontic brackets and tubes requires roughening of the surface for mechanical interlocking between the surface and the adhesive for enamel and composite surfaces. For ceramics, a combination of mechanical and chemical bonding is needed. Ideally, only the surface where the bracket will be bonded should be conditioned. In this study, we attempted to condition a limited surface for bracket placement (about $1 \mathrm{~mm}$ extra around the planned position of the bracket). We determined that the different methods used to increase the roughness of enamel, composite and ceramic dental surfaces led to differences in roughness, depending on the method used and the underlying surface of the tooth. Roughening of the enamel was accomplished by covering the area where the bracket would be bonded with etching gel (35\% phosphoric acid) for about 10 seconds. With etching, a small amount of the interprismatic enamel is removed to enable penetration of the adhesive into small holes. The prismatic part of the enamel will remain; while, the adhesive will penetrate the resolved interprismatic areas. If a carborundum bur or a sandblaster is used to roughen the surface, the total surface of the tooth is roughened. We found the roughness of enamel surfaces was significantly increased when a bur was used compared with a sandblaster or an etching procedure with phosphoric acid. For composites, there was a significant increase in surface roughness when a sandblaster was used compared with a bur. For ceramic surfaces, the roughness was significantly higher after sandblasting compared with etching with hydrochloric acid for 120 seconds. We found the mean shear bond strength was significantly ( $\mathrm{p}<$ 0.01 ) higher for all tested surfaces when they were less rough. Thus, orthodontists can use the most conservative surface treatment for accurate bonding of brackets: etching with phosphoric acid for enamel, roughening of composite with a bur, and etching with hydrofluoric acid for ceramic surfaces. There was a relatively large standard deviation found in the mean values of shear bond strength and the adhesive remnant index in this in vitro study (Figures 2 and 3 ); thus, it can be expected that even if proper surface preparation is applied, inadequate bonding ( $<5.7 \mathrm{MPa}$ or $>8 \mathrm{MPa}$ values) will occur.

We also found enamel prepared with a bur or sandblaster required cleaning of the surface and etching with phosphoric acid for accurate bonding. Adequate bond strength was also found for bonding on enamel after etching with phosphoric acid only. There was no significant difference in shear bond strength between uncoated and precoated brackets bonded on enamel after etching with phosphoric acid. For composites, surface preparation with a bur was sufficient for adequate bond values.

For ceramic surfaces, a bur was not used for surface preparation because permanent damage was expected. Using a sandblaster and ceramic activator for preparation of ceramic surfaces did not lead to adequate bond strength. Thus, only etching with hydrofluoric acid and the application of a ceramic activator is applicable for preparing 
ceramic surfaces. It should be noted that all brackets bonded on ceramic without using a porcelain activator (salinization) were lost during thermo cycling. Thus, mechanical bonding is not sufficient for ceramic surfaces. Chemical bonding is only achieved after applying a ceramic activator. In this study, we did not found significant differences in mean shear bond strengths or ARI scores between precoated and non-precoated brackets except after sandblasting composite surfaces.

After curing, the brackets and tubes should be used for orthodontic tooth movement. If light curing is used, the wires can be inserted immediately after curing. In vivo, saliva will always be present during orthodontic treatments and oral environment changes caused by drinks and food will lead to temperature changes. This could cause differences in the bonding capacity of the adhesive. In vivo, the presence of saliva and temperature changes in the oral environment will eventually cause degradation of the interface between the dental surface and the adhesive [14]. In this in vitro study, we found no statistically significant difference between MPa values for samples with and without thermo cycling; thus, it can be expected that the results could be confirmed in an in vivo experiment.

After removal of the brackets, the remaining adhesive should be removed from the tooth surface and the surfaces should be restored to an esthetically pleasing and easy to clean tooth surface. As expected, we found (as presented in Figure 3) a relatively high ARI score for bonding methods with relatively high shear bond strengths. When the ARI scores of different surface preparation procedures for enamel were compared, we found using a bur or a sandblaster did not lead to significant differences. However, after etching of the enamel surface with phosphoric acid, a significantly $(\mathrm{p}<0.01)$ higher ARI-score was found. Also for composite surfaces, no significant differences in ARI scores were found if a bur or a sandblaster was used for preparation. The ARI score for ceramic surfaces after etching with hydrofluoric acid was significantly higher compared with preparation with a sandblaster.

The use of a bur or sandblaster before etching enamel led to an increased amount of residual adhesive on the enamel after debonding and removal can lead to surface scratches, cracking, and loss of sound enamel. Conversely, less residual adhesive was present after less accurate bonding. This could be clinically advantageous because when brackets fail at the enamel-adhesive interface, less adhesive remains and tooth cleanup is likely to be easier and faster. The ARI scores for composites were significantly lower compared with the ARI scores after debonding brackets from enamel surfaces. After debonding of brackets from the ceramic surface, almost all adhesive remained. Therefore, the cleanup for ceramic surfaces after debonding will take more time [16]. After surface preparation with etching, a drill, or sandblasting, a primer was used in this study for enamel and composite surfaces. Self-etching primers (SEPs) have been introduced $[17,18]$ that combine the etching and applying of a primer procedures for enamel preparation. The use of SEPs could simplify the bonding process and reduce the time for bonding orthodontic brackets and tubes by reducing the steps needed. The difference between the overall bond failure rate and the mean bond failure rate per patient for a twostep (etching and primer) surface preparation vs. SEPs was not statistically significant [18]. These SEPs were not used in this study because the roughness of the surface was tested before applying a primer.

The purpose of this in vitro study was to test an optimal protocol for bonding orthodontic brackets on various dental surfaces. We found that for enamel, the traditional approach of etching with a $35 \%$ phosphoric acid gel for 10 seconds and application of a primer led to accurate bonding of an orthodontic bracket. For bonding orthodontic brackets onto composite surfaces, roughening of the surface with a drill and cleaning with water and air spray, followed by drying of the surface and application of a primer seems to be sufficient for effective bonding of brackets. For bonding on ceramic surfaces, surface preparation using etching with hydrofluoric acid to roughen the ceramic surface for a mechanical retention followed by application of a thin layer of a silane-coupling agent (porcelain activator) was needed for a chemical bonding between the adhesive and the ceramic surface.

It was expected that an increased roughness of the dental surface should increase the mechanical interlocking between the dental surface and the adhesive. In this study, we found an increase in surface roughness did not lead to an increase in bond strength. As permanent damage caused by the surface treatment required for bonding should be avoided, it can be concluded that roughening of the dental surface should and can be reduced without reduction of bonding efficiency. Studies evaluating differences in the efficiency of non-precoated and precoated brackets had different outcomes $[18,19]$. Theoretically, the placement of adhesive in the factory precoating should result in an effective connection between the bracket mesh and reduce bracket failures. In this study, no significant differences in bonding strength were found between these two groups, except when bonding brackets on a sandblasted composite surface. In general, the advantages of precoated brackets such as increased bonding efficiency, shorter bonding times, and less bond failures should be evaluated. The use of extracted premolars as tooth surfaces for bonding orthodontic brackets could have introduced some variables, such as the fluoride concentration in the enamel and the storage of the tooth after extraction at the start of the study [20,21]. Finally, the results of this study are only applicable for the materials and methods used.

\section{Conclusions}

In this in vivo study using selected materials, we found the following: Thermo cycling of teeth does not result in differences in bond strength. A significant increase in bonding strength between brackets without adhesive and precoated brackets was only found after bonding on sandblasted composite surfaces. The increased roughness of dental surfaces did not result in increased bonding strength. There was adequate bonding of metal brackets on enamel after etching with a $37 \%$ phosphoric acid for 30 seconds. If enamel surfaces are roughened with a drill or sandblaster before etching, a too high shear bonding strength over $8 \mathrm{MPa}$ can be expected. Even if the surface is properly prepared, inadequate bonding (too high or too low MPa values) will occur.

Just roughening of the enamel surface will not lead to efficient bonding; although, roughening of a composite surface with a bur will. Sandblasting of composite surfaces will usually not lead to adequate bonding, as etching with a hydrofluoric acid solution along with a porcelain activator is needed. A reduction in surface roughness will lead to an increase in bond strength. After debonding, the quantity of remaining adhesive depends on the surface properties and the surface preparation before bonding. 
Citation: Breuning H, Dekkers L, Beeftink M, Schols J, Wolke J (2014) Bonding Metal Brackets on Tooth Surfaces. Dentistry 4: 231. doi:

Page 6 of 6

\section{Acknowledgements}

The authors are thankful for the brackets, etching, primers, activator, and adhesive, which were provided by the $3 \mathrm{M} / \mathrm{Unitek}$ Company.

\section{References}

1. Reynolds IR, von Fraunhofer JA (1976) Direct bonding of orthodontic brackets-a comparative study of adhesives. Br J Orthod 3: 143-146.

2. Powers JM, Kim H-B, Turner DS (1997) Orthodontic adhesives and bond strength testing. Semin Orthod 3: 147-156.

3. Olsen EM, Bishara SE, Damon P, Jakobsen JR (1997) Comparison of shear bond strength and surface structure between conventional acid etching and air-abrasion of human enamel. Am J Orthod Dentofacial Orthop 112: 502-506.

4. Eslamian L, Borzabadi-Farahani A, Mousavi N, Ghasemi A (2011) The effects of various surface treatments on the shear bond strengths of stainless steel brackets to artificially-aged composite restorations. Austr Orthod J 27: 28-32.

5. Trakyali G, Malkondu Ö, Kazazoğlu E, Arun T (2009) Effects of different silanes and acid concentrations on bond strength of brackets to porcelain surfaces. Eur J Orthod 31: 402-406.

6. Schmage P, Nergiz I, Herrmann W, Özcan M (2003) Influence of various surface-conditioning methods on the bond strength of metal brackets to ceramic surfaces. Am j Orthod Dentofacial Orthod 123: 540-546.

7. Wong M, Power S (2003) A prospective randomized clinical trial to compare pre-coated and non-pre-coated brackets. J Orthod 30: 155-158.

8. Bishara SE, Oonsombat C, Soliman MM, Warren JJ, Laffoon JF, et al. (2005) Comparison of bonding time and shear bond strength between a conventional and new integrated bonding system. Angle Orthod 75: 237-242.

9. Hajrassie MK, Khier SE (2007) In-vivo and vitro comparison of bond strength of orthodontic brackets bonded to enamel and debonded at various times. Am J Orthod Dentofacial Orthop 131: 384-390.
10. Maurya R, Tripathi T, Rai P (2011) New generation of color bonding: A comparative in vitro study. Ind J Dent Res 2: 733-734.

11. Årtun J, Bergland S (1984) Clinical trials with crystal growth conditioning as an alternative to acid-etch enamel pretreatment. Am J Orthod 85: 333-340.

12. Tecco S, Trainini T, Caputi S, Festa F, de Luca V, et al. (2005) A new onestep dental flowable composite for orthodontic use: an in vitro bond strength study. Angle Orthod 75: 672-677.

13. Lindauer SJ, Browning H, Shroff B, Marshall F, Anderson RH, et al. (1997) Effect of pumice prophylaxis on the bond strength of orthodontic brackets. Am J Orthod Dentofacial Orthop 11: 599-603.

14. Barry GR (1995) A clinical investigation of the effects of omission of pumice profylaxis on band and bond failure. Br J Orthod 22: 245-248.

15. Ireland AJ, Sherriff $M(2002)$ The effect of pumicing on the in vivo use of a resin-modified glass poly (alienates) cement and a conventional no-mix composite for bonding orthodontic brackets. J Orthod 29: 217-220.

16. Khoroushi M, Motamedi SH (2007) Shear bond strength of compositeresin to porcelain: effect of thermocycling. J Dent 4: 21-26.

17. Ajlouni R, Bishara SE, Oonsombat C, Denehy GE (2005) Evaluation of modifying the bonding protocol of a new acid-etch primer on the shear bond strength of orthodontic brackets. Angle Orthod 74: 410-413.

18. Rajagopal R, Padmanabhan S, Gnanamani J (2004) A comparison of shear bond strength and debonding characteristics of conventional and bonding-insensitive and self-etching primers in vitro. Angle Orthod 74: 264-268.

19. Vincente A, Bravo LA (2007) Shear bond strength of precoated and uncoated brackets, using a self-etching primer. Angle Orthod 77: 524-527.

20. Bishara SE, Olsen M, Von Wald L (1997) Comparison of shear bond strength of precoated and uncoated brackets. Am J Orthod 112: 617-621.

21. Leódido Gda R, Fernandes HO, Tonetto MR, Presoto CD, Bandéca MC, et al. (2012) Effect of fluoride solutions on the shear bond strength of orthodontic brackets. Braz Dent J 23: 698-702. 